George Z. Voyiadjis* and Peter I. Kattan

\title{
On certain fundamental issues in continuum damage mechanics
}

\begin{abstract}
In this article, we discuss three fundamental issues in continuum damage mechanics. First, we investigate the nature of the damage process. For this purpose, we dissect the expression of the effective stress into an infinite geometric series and introduce several stages of damage that we call primary damage, secondary damage, tertiary damage, etc. The second issue to be discussed is the problem of small damage. In this regard, we introduce a new definition of the damage variable that is suitable for small-damage cases. Finally, we discuss the new concept of undamageable materials. These are currently hypothetical materials that maintain a zero value of the damage variable throughout the deformation process. It is hoped that these proposed new types of materials will open the way to new areas of research in both damage mechanics and materials science.
\end{abstract}

Keywords: damage; damage mechanics; small damage; undamageable; undamageable material.

*Corresponding author: George Z. Voyiadjis, Department of Civil and Environmental Engineering, Louisiana State University, Baton Rouge, LA 70803, USA, e-mail: voyiadjis@eng.Isu.edu

Peter I. Kattan: Department of Civil and Environmental Engineering, Louisiana State University, Baton Rouge, LA 70803, USA

\section{The nature of the damage process}

In this section, we investigate the nature of the damage process within the framework of continuum damage mechanics [1-27]. For this purpose, we will dissect the expression of the effective stress into an infinite geometric series. Consider the infinite geometric series as follows:

$$
a r+a r^{2}+a r^{3}+\ldots=\frac{a}{1-r}
$$

The geometric series of Eq. (1) is valid for $|\mathrm{r}|<1$. Consider now the effective stress given by

$$
\bar{\sigma}=\frac{\sigma}{1-\varphi}
$$

The expression of the effective stress given in Eq. (2) is the classical expression taken from the theory of continuum damage mechanics [1-27]. In expression (2), $\varphi$ is the damage variable (its values lie between 0 and 1), $\sigma$ is the Cauchy stress, and $\bar{\sigma}$ is the corresponding effective stress. Looking at the right-hand side of the geometric series of Eq. (1), we can see that the expression of the effective stress of Eq. (2) is the sum of the infinite geometric series. It satisfies the condition because $0<\varphi<1$.

Thus, the effective stress of Eq. (2) can be written by making an analogy with the infinite geometric series of Eq. (1) as follows:

$$
\bar{\sigma}=\sigma\left(1+\phi+\phi^{2}+\phi^{3}+\ldots\right)
$$

Eq. (3) is an infinite exact relationship. To interpret relation (3) physically, we can say that the damage process consists of an infinite number of smaller damage processes or stages. Because we have $\bar{\sigma} \bar{A}=\sigma A$, where $A$ is the cross-sectional area and $\bar{A}$ is the effective cross-sectional area (the cross-sectional area in the fictitious effective configuration), we can rewrite Eq. (3) as follows:

$$
\frac{A}{\bar{A}}=1+\phi+\phi^{2}+\phi^{3}+\ldots
$$

Based on Eq. (4), we can say that the damage process consists of several smaller damage processes or stages: the primary damage stage (when we take the first two terms of the series), the secondary damage stage (when we take the third term of the series), and the tertiary damage stage (when we take the fourth term of the series). We can continue in an infinite number of smaller and smaller damage stages. However, for practical purposes, it suffices to consider the first four terms of the infinite geometric series.

\subsection{Primary damage variable}

Consider the first two terms of the series in Eq. (4) to define the primary damage variable as follows:

$$
\frac{A}{\bar{A}}=1+\varphi_{p}
$$


We can solve Eq. (5) to obtain the following explicit expression for the primary damage variable:

$$
\varphi_{p}=\frac{A}{\bar{A}}-1
$$

\subsection{Secondary damage variable}

Consider the first three terms of the series in Eq. (4) and define the secondary damage variable as follows:

$$
\frac{A}{\bar{A}}=1+\varphi_{s}+\varphi_{s}^{2}
$$

We can solve the quadratic equation [Eq. (7)] to obtain the following explicit expression for the secondary damage variable:

$$
\varphi_{s}=-\frac{1}{2}+\frac{1}{2} \sqrt{-3+4 \frac{A}{\bar{A}}}
$$

\subsection{Tertiary damage variable}

Consider the first four terms of the series in Eq. (4) and define the tertiary damage variable as follows:

$$
\frac{A}{\bar{A}}=1+\varphi_{t}+\varphi_{t}^{2}+\varphi_{t}^{3}
$$

We can solve the cubic equation [Eq. (7)] to obtain the following explicit expression for the tertiary damage variable:

$$
\varphi_{t}=-\frac{1}{3}+\frac{1}{6} \sqrt[3]{-80+108 \frac{A}{\bar{A}}+12 \sqrt{48-120 \frac{A}{\bar{A}}+81\left(\frac{A}{\bar{A}}\right)^{2}}}
$$

Thus, we have established the explicit expressions for the damage variables at the primary, secondary, and tertiary damage stages. In this work, we present the mathematical formulation of this dissection of the damage process into the three stages. However, we leave the physical interpretation of this dissection process to future work.

\section{Small-damage processes}

In this section, we present some observations on the problem of small-damage processes. In [28], the physics of phase fields was used to derive the following generalized relationship between the Cauchy stress and the effective stress:

$$
\bar{\sigma}=\frac{\sigma}{(1-\varphi) \sqrt{2 \varphi+1}}
$$

Expression (11) should be compared with the classical expression given in Eq. (2). It should be noted that the above expression for the effective stress corresponds to a cubic formula for $\varphi$ in terms of areas. The reader is referred to reference [28] for more details.

Consider the square root term that appears in the denominator of Eq. (11). We can use the Taylor series expansion and take the first two terms of the Taylor series expansion of the square root function to obtain the following approximation

$$
\sqrt{2 \varphi+1} \approx 1+\varphi
$$

for small values of $\varphi$, i.e., for small damage. Therefore, we can write the following expression for the effective stress of Eq. (11) in the case of small damage:

$$
\bar{\sigma}=\frac{\sigma}{(1-\phi)(1+\phi)}=\frac{\sigma}{1-\phi^{2}}
$$

which applies for the case of small damage. Formula (13) corresponds to the damage variable $\varphi=\sqrt{\frac{A-\bar{A}}{A}}$ compared with $\varphi=\frac{A-\bar{A}}{A}$ in the classical case.

Alternatively, we can postulate the following expression for the effective stress in the case of large damage:

$$
\bar{\sigma}=\frac{\sigma}{1-\sqrt{\phi}},
$$

which corresponds to the damage variable $\varphi=\left(\frac{A-\bar{A}}{A}\right)^{2}$.

We can generalize the above two expressions for the effective stress of Eqs. (13) and (14) when the exponent is increased from 2 to $3,4, \ldots, n$, where $n$ approaches infinity. We propose the following generalized definitions:

$$
\bar{\sigma}=\frac{\sigma}{1-\varphi^{n}} \quad \text { for small damage }
$$




$$
\bar{\sigma}=\frac{\sigma}{1-\varphi^{1 / n}} \quad \text { for large damage }
$$

Use $n=1$ for normal (intermediate) damage.

\section{The concept of undamageable materials}

In this section, we elaborate a little on the new concept of undamageable materials. These hypothetical materials were proposed recently by the authors [29-31]. These types of material compare with rubber materials [32]. The authors also made comparisons between undamageable materials and various nonlinear elastic materials taken from the book of Bower [33].

Undamageable materials are proposed and designed in such a way so as to maintain a zero value for the damage variable throughout the deformation process. This formulation was presented by the authors in great detail [29-31] within the concept of continuum damage mechanics. Thus, it can be seen that undamageable materials are desirable because they cannot be damaged at all.
It is hoped that the manufacturing technology will reach a stage in the future where the realization of this type of material can be achieved.

To show that such materials maintain a zero value of the damage variable throughout the deformation process, we need to modify the classical definition of the effective stress of Eq. (2) to become as follows:

$$
\bar{\sigma}=\frac{\sigma}{\sqrt[n]{1-\varphi}}
$$

We can now perform the following derivation when $n$ approaches infinity:

$$
\bar{\sigma}=\frac{\sigma}{\sqrt[n]{1-\varphi}}=\frac{\sigma}{(1-\varphi)^{1 / n}}=\frac{\sigma}{(1-\varphi)^{1 / \infty}}=\frac{\sigma}{(1-\varphi)^{0}}=\frac{\sigma}{1}=\sigma
$$

Thus, we obtain the undamageable material in this case. In our previous publications [29-31], we presented the concept of undamageable materials using the definition of the damage variable in terms of elastic stiffness degradation. We have now supported that formulation by presenting the concept of undamageable materials using a slightly modified form of the effective stress based on the cross-sectional area reduction as shown in Eqs. (17) and (18).

\section{References}

[1] Celentano DJ, Tapia PE, Chaboche, J-L. In Mecanica Computacional, Vol. XXIII. Buscaglia G, Dari E, Zamonsky O, Eds., Bariloche, Argentina, 2004.

[2] Doghri I. Mechanics of Deformable Solids: Linear and Nonlinear, Analytical and Computational Aspects. SpringerVerlag: Berlin, 2000.

[3] Hansen NR, Schreyer HL. Int. J. Solids Struct. 1994, 31, 359-389.

[4] Kachanov L. Izv. Akad. Nauk. USSR Otd. Tech. 1958, 8, 26-31 [in Russian].

[5] Kattan PI, Voyiadjis GZ. Int. J. Eng. Sci. 1990, 28, 421-435.

[6] Kattan PI, Voyiadjis GZ. Int. J. Eng. Sci. 1993, 31, 183-199.

[7] Kattan PI, Voyiadjis GZ. J. Eng. Mech. ASCE 2001, 127, 940-944.

[8] Kattan PI, Voyiadjis GZ. Damage Mechanics with Finite Elements: Practical Applications with Computer Tools. SpringerVerlag: Berlin, 2001.

[9] Ladeveze P, Poss M, Proslier L. Proceedings of the Fourth International Conference on Composite Materials, Japan Society for Composite Materials, Vol. 1, 1982, pp. 649-658.

[10] Ladeveze P, Lemaitre J. The 16th International Congress of Theoretical and Applied Mechanics, Lyngby, Denmark, 1984.

[11] Lubineau G. Int. J. Damage Mech. 2010, 19, 499-518.

[12] Lubineau G, Ladeveze P. Comput. Mater. Sci. 2008, 43, 137-145.

[13] Lee H, Peng K, Wang J. Eng. Fracture Mech. 1985, 21, 1031-1054.
[14] Luccioni B, Oller S. Comput. Methods Appl. Mech. Eng. 2003, 192, 1119-1145.

[15] Nichols JM, Abell AB. North American Masonry Conference, Clemson, SC, 2003.

[16] Nichols JM, Totoev YZ. North American Masonry Conference, Austin, TX, 1999.

[17] Rabotnov Y. Proceedings of the Twelfth International Congress of Applied Mechanics, Stanford, 1968. Hetenyi M, Vincenti WG, Eds., Springer-Verlag: Berlin, 1969, pp. 342-349.

[18] Rice JR. J. Mech. Phys. Solids 1971, 19, 433-455.

[19] Sidoroff F. IUTAM Colloqium on Physical Nonlinearities in Structural Analysis. Springer-Verlag: Berlin, 1981, pp. 237-244.

[20] Voyiadjis GZ. Int. J. Plast. 1988, 4, 335-353.

[21] Voyiadjis GZ, Kattan PI. Int. J. Eng. Sci. 1990, 28, 505-524.

[22] Voyiadjis GZ, Kattan PI. Int. J. Eng. Sci. 1992, 30, 1089-1108.

[23] Voyiadjis GZ, Kattan PI. Damage Mechanics. Taylor \& Francis/ CRC Press, 2005.

[24] Voyiadjis GZ, Kattan PI. Advances in Damage Mechanics: Metals and Metal Matrix Composites with an Introduction to Fabric Tensors, 2nd ed. Elsevier: Oxford, UK, 2006.

[25] Voyiadjis GZ, Kattan PI. J. Mech. Behav. Mater. 2006, 17, 31-56.

[26] Voyiadjis GZ, Kattan PI. Mech. Adv. Mater. Struct. 2006, 13, 285-301.

[27] Voyiadjis GZ, Kattan PI. Int. J. Damage Mech. 2009, 18, $315-340$. 
[28] Voyiadjis GZ, Mozaffari N. Int. J. Solids Struct. (under review).

[29] Voyiadjis GZ, Kattan PI. ASME J. Mater. Technol. 2012, 134, 02101.

[30] Voyiadjis GZ, Kattan PI. Int. J. Damage Mech. 2013, doi: 10.1177/1056789512446518 [Epub ahead of print].
[31] Voyiadjis GZ, Kattan PI. ASME J. Eng. Mat. Tech. 2013 (accepted for publication).

[32] Arruda EM, Boyce MC. J. Mech. Phys. Solids 1993, 41, 389-412.

[33] Bower AF. Advanced Mechanics of Solids. CRC Press: Boca Raton, FL, 2009. 